\title{
Studies on the purification of polysaccharides separated from Tremella fuciformis and their neuroprotective effect
}

\author{
YAXIANG JIN $^{1}$, XINYU HU ${ }^{2}$, YAN ZHANG ${ }^{3}$ and TIANJI LIU ${ }^{1}$ \\ ${ }^{1}$ Physical Examination Center, China-Japan Union Hospital of Jilin University, Changchun, Jilin 130033; \\ ${ }^{2}$ Department of Clinical Medicine, Changchun Medical College, Changchun, Jilin 130021; ${ }^{3}$ Department of Breast Surgery, \\ The Second Hospital of Jilin University, Changchun, Jilin 130041, P.R. China
}

Received April 29, 2015; Accepted March 4, 2016

DOI: $10.3892 / \mathrm{mmr} .2016 .5026$

\begin{abstract}
The present study aimed to investigate the protective effect of purified polysaccharides from Tremella fuciformis against glutamate-induced cytotoxicity in differentiated PC12 (DPC12) cells. The aqueous extract of Tremella fuciformis was purified using a DEAE-52 cellulose anion exchange column and a Sepharose G-100 column, respectively. A fraction termed TL04 with a 2,033 kDa molecular weight was obtained. The backbone of TL04 is composed of $(1 \rightarrow 2)$ - and $(1 \rightarrow 4)$-linked-mannose and $(1 \rightarrow 3)$-linked-glucans. Results revealed that TL04 treatment improved cell viability and suppressed reactive oxygen species accumulation, lactose dehydrogenase release and caspase- 3 activity, and ameliorated mitochondrial abnormal alteration caused by glutamate. TL04 pretreatment enhanced the level of B-cell lymphoma 2 (Bcl-2), and suppressed Bax expression and cytochrome $c$ (Cyto C) release in glutamate-treated cells. Exposure to glutamate strongly increased the activity of caspase- 8 , caspase- 9 and caspase- 3 , which were significantly reversed by TL04 pretreatment. The presence of Ac-DEVD-CHO (a caspase-3 inhibitor) markedly enhanced the potency of TL04 in improving the viability of glutamate-exposed DPC12 cells. Collectively, the results demonstrated that the purified polysaccharides separated from Tremella fuciformis (TL04) possess a neuroprotective effect against glutamate-induced DPC12 cell damage predominantly through the caspase-dependent mitochondrial pathway. The present study provides an experimental foundation supporting purified TL04 as a potential therapeutic agent for neurodegenerative diseases.
\end{abstract}

Correspondence to: Professor Tianji Liu, Physical Examination Center, China-Japan Union Hospital of Jilin University, Building 3, 126 Xiantai Street, Changchun, Jilin 130033, P.R. China

E-mail: liutj1000@163.com

Key words: Tremella fuciformis, purified polysaccharides, glutamate, neuroprotection, mitochondria

\section{Introduction}

Glutamate, a fast excitatory neurotransmitter in the central nervous system (1), is also responsible for neurotoxicity via excitotoxicity and oxidative pathways $(2,3)$. As reported previously, excessive release of glutamate leads to acute and chronic brain diseases including brain ischemia, traumatic brain injury, and neurodegenerative disorders $(4,5)$. Several signaling pathways participate in glutamate-induced neurotoxicity, one of which is associated with the mitochondria (6). B-cell lymphoma 2 (Bcl-2) and Bax, located in the mitochondria, are considered to be associated with mitochondrial function (7). As reported by a previous study, reactive oxygen species (ROS), a byproduct of cellular oxidative processes, is responsible for mitochondrial depolarization (8). In addition, enhanced mitochondrial permeabilization causes intracellular ROS accumulation, which further results in mitochondria dysfunction $(9,10)$. Experiments demonstrate that the reduction of mitochondrial membrane potential (MMP, $\Delta \psi \mathrm{m}$ ) leads to the release of cytochrome $c$ (Cyto C), whose cytoplasmic localization initiates caspase-dependent apoptotic cell death.

Due to the various pharmacological functions of polysaccharides, the natural purified polysaccharides are widely used in the food and pharmaceutical industries. It was confirmed that the chemical composition, glycosidic linkages, conformation, molecular weight, and degree of branching of polysaccharides were associated with their bioactivities (11). As potent macrofungi-derived substances, polysaccharides separated from Cordyceps militaris (12), Ganoderma lucidum (13), and Tricholoma matsutake (14) have been extensively investigated. However, limited work has been conducted on the purification and bioactivities of polysaccharides separated from Tremella fuciformis.

Tremella fuciformis, known as a nutritious mushroom, is popular in China as a medicinal remedy with tonic actions for treating debility and exhaustion. As one of the primary active components, polysaccharides obtained from the Tremella fuciformis are reported to possess immunomodulatory (15), anticancer (16), and anti-inflammatory activities (17). Tremella fuciformis successfully enhances the neurite outgrowth of PC12 cells and restores trimethyltin-induced impairment of memory in rats (18). Polysaccharides isolated from Tremella fuciformis 
exhibits a protective effect against radiation-induced damage in mice (19). It is also reported that the purified Tremella fuciformis polysaccharides possess anti-oxidant activity (20). All these data indicate that polysaccharides isolated from Tremellafuciformis may exhibit a neuroprotective function.

In the present study, polysaccharides extracted from Tremella fuctiformis were purified and characterized. The neuroprotective effect of purified polysaccharides on glutamate induced differentiated PC12 (DPC12) cell damage and the underlying mechanisms were investigated. The data revealed that the purified polysaccharides improved cell viability and mitochondrial function, and restored the abnormal expression of apoptosis-related proteins. All the findings demonstrated that mitochondria-related pathways are essential for neuroprotection against glutamate-induced toxicity in DPC12 cells.

\section{Materials and methods}

Crude extract preparation and preliminary identification. As demonstrated in a previous study (21), $100 \mathrm{~g}$ Tremella fuciformis powder was extracted twice using $90^{\circ} \mathrm{C}$ water for $2.5 \mathrm{~h}$. After centrifuging at $2667 \mathrm{x}$ g for $10 \mathrm{~min}$, the supernatant was sequentially concentrated and freeze-dried for further experiments.

Purification of Tremella fuciformis polysaccharides. The protein in Tremella fuciformis water extract was removed using Sevag reagent [V (n-butanol): V (chloroform)=1:4, $50 \mathrm{ml}$ ] (22). After adding 4X ethanol, the precipitation was dissolved in double distilled (dd) $\mathrm{H}_{2} \mathrm{O}$ and subjected to the DEAE-52 cellulose anion exchange column $(2.6 \times 35 \mathrm{~cm}$; Whatman; GE Healthcare Life Sciences, Chalfont, UK). The column was eluted with $\mathrm{ddH}_{2} \mathrm{O}$ followed by $0.1 \mathrm{~mol} / \mathrm{l}$ and $0.3 \mathrm{~mol} / 1 \mathrm{NaCl}$ at a flow rate of $1 \mathrm{ml} / \mathrm{min}$. Collected polysaccharides were further purified using a gel permeation chromatography system Sepharose G-100 (Pfizer, New York City, NY, USA). The column was eluted with $\mathrm{ddH}_{2} \mathrm{O}$ at a flow rate of $0.4 \mathrm{ml} / \mathrm{min}$. The fractions $(20 \mathrm{ml})$ were collected.

Cellular morphology analysis. PC12 cells (1x105), obtained from the American Type Culture Collection, were seeded into each well in a six-well plate. After differentiation, cells were pre-treated with 5 and $20 \mathrm{mg}$ TL0 4 for $3 \mathrm{~h}$, followed with $12 \mathrm{~h}$ co-incubation with $20 \mathrm{mM}$ L-Glu (Beijing Dingguo Tech Changseng Biotechnology Co., Ltd., Beijing, China). Then, cells were incubated with Hoechst $33342(5 \mathrm{mg} / \mathrm{mL}$; Sigma-Aldrich, St. Louis, MO, USA) for $15 \mathrm{~min}$ at $37^{\circ} \mathrm{C}$ in darkness. The fluorescence intensity in the nucleus was photographed using a fluorescent microscope (x20 objective; CCD camera, Nikon Corporation, Tokyo, Japan) after being washed with phosphate buffered saline 3 times.

Fouriertransforminfraredspectroscopy (FTIR)determination . In total, a $4 \mathrm{mg}$ sample was ground thoroughly with $150 \mathrm{mg}$ $\mathrm{KBr}$ into a smooth mortar. The average transmission spectra $(n=100)$ were recorded via an IRPrestige-21 FTIR spectrometer (Shimadzu, Tokyo, Japan) at a wavelength ranging from 400 to $4,000 \mathrm{~cm}^{-1}$.
Homogeneity and molecular weight determination. The homogeneity and molecular weight were analyzed by LC-10ATvp high performance liquid chromatography (HPLC) system (Shimadzu, Tokyo, Japan) equipped with a TSK-GEL G4000PWXL column (Tosoh Co., Tokyo, Japan) and an Alltech 2000ES ELSD (Shimadzu, Tokyo, Japan). Similar to a previous study (23), dd $\mathrm{d}_{2} \mathrm{O}$ served as the mobile phase, the flow rate was $0.45 \mathrm{ml} / \mathrm{min}$, aerosol level was $60 \%$, drift tube temperature was $120^{\circ} \mathrm{C}$ and nebulising nitrogen pressure was 25 psi. The dextran standards were used to create a calibration curve.

Periodate oxidation-smith degradation reaction of polysaccharides. Similar to a previous study (23), $20 \mathrm{mg}$ polysaccharide was dissolved in $15 \mathrm{mM} \mathrm{NaIO}_{4}(25 \mathrm{ml}, \mathrm{pH} 4)$ in darkness at $4^{\circ} \mathrm{C}$. $\mathrm{HIO}_{4}$ consumption was calculated and the formic acid production was determined by titration. After 48-h dialyzing against $\mathrm{ddH}_{2} \mathrm{O}$, the dialysate was concentrated and reduced with potassium borohydride $(70 \mathrm{mg})$ overnight at room temperature. Adjusting to $\mathrm{pH} 7.0$ by addition of acetic acid, the solution was dialyzed against $\mathrm{ddH}_{2} \mathrm{O}$ for another $24 \mathrm{~h}$. Then, $3 \mathrm{ml}$ of sample was detected by the HPLC/ELSD system. The rest of the product was hydrolyzed with $1 \mathrm{M} \mathrm{H}_{2} \mathrm{SO}_{4}$ at $25^{\circ} \mathrm{C}$ for $40 \mathrm{~h}$, and adjusted to $\mathrm{pH} 7.0$ by $\mathrm{BaCO}_{3}$. The solution was centrifuged at $2134 \mathrm{x}$ g for $10 \mathrm{~min}$ to separate the hydrolysates, which were further analyzed by HPLC/ELSD.

Cell culture. PC12 cells, were grown in Dulbecco's modified Eagle's medium (DMEM) supplemented with $10 \%$ horse serum (HS), $5 \%$ fetal bovine serum (FBS), penicillin (100 U/ml) and streptomycin $(100 \mu \mathrm{g} / \mathrm{ml})$ (all from Thermo Fisher Scientific, Inc.), under a humidified atmosphere containing $5 / 95 \% \mathrm{CO}_{2} /$ air at $37^{\circ} \mathrm{C}$. The culture medium was changed every 3 days. PC12 cells were differentiated for $48 \mathrm{~h}$ with $20 \mathrm{ng} / \mathrm{ml}$ nerve growth factor dissolved in DMEM medium containing $1 \% \mathrm{FBS}$ and $1 \% \mathrm{HS}$.

Cell viability analysis. As reported previously, a quantitative colorimetric assay with 3-(4,5-cimethylthiazol-2-yl)-2,5-diphenyl tetrazolium bromide (MTT; Sigma-Aldrich) was applied to measure cell viability (24). Differentiated PC12 cells were seeded into 96-well plates at $1 \times 10^{4}$ cells/well. Cells were treated with TL04 (5 and $20 \mu \mathrm{g}$ ) alone for $24 \mathrm{~h}$, or pretreated with TL04 (5 and $20 \mu \mathrm{g}$ ) for $3 \mathrm{~h}$ and exposed to $20 \mathrm{mM}$ glutamate for another $24 \mathrm{~h}$. In another separated experiment, cells were pretreated with $10 \mu \mathrm{M}$ Ac-DEVD-CHO for $30 \mathrm{~min}$, and exposed to $5 \mu \mathrm{g}$ and $20 \mu \mathrm{g}$ TL04 for $3 \mathrm{~h}$, followed by another $24 \mathrm{~h}$ co-incubation with $20 \mathrm{mM}$ glutamate. After incubation with $0.5 \mathrm{mg} / \mathrm{ml} \mathrm{MTT} \mathrm{solution} \mathrm{for} 4 \mathrm{~h}$ at $37^{\circ} \mathrm{C}$ in darkness, $100 \mu 1$ dimethyl sulfoxide was added to dissolve crystals. A microplate reader (Bio-Rad Laboratories, Inc., Hercules, CA, USA) was used to detect the absorbance at $540 \mathrm{~nm}$. Viability values of treated cells were expressed as a percentage of that from corresponding control cells.

Mitochondrial membrane potential (MMP) analysis. PC12 cells $\left(1 \times 10^{5}\right)$ were seeded into each well of a 6 -well plate. After differentiation, cells were pre-treated with $5 \mu \mathrm{g}$ and $20 \mu \mathrm{g}$ TL04 for $3 \mathrm{~h}$, followed by 12-h co-incubation with $20 \mathrm{mM}$ 
glutamate. The changes in MMP were measured via 5,5', 6,6'-tetrachloro-1,1',3,3' tetraethylbenzimidazolylcarbocyanine iodide (JC-1; Sigma-Aldrich) staining. Treated cells were incubated with $2 \mu \mathrm{M} \mathrm{JC}-1$ at $37^{\circ} \mathrm{C}$ for $10 \mathrm{~min}$. Fluorescent microscope (20x objective; Axio Observer Z1, CCD camera; Carl Zeiss, Germany) was applied to record the fluorescent color in each group. Average ratio of red (590 nm; healthy cells) to green $(540 \mathrm{~nm}$; apoptotic or unhealthy cells) fluorescence intensity of each cell was calculated by ImageJ software ( $n=50$; National Institutes of Health, Bethseda, MA, USA). Values of treated cells were expressed as a percentage of that from corresponding control cells.

Intracellular lactate dehydrogenase $(L D H)$ release, $R O S$ accumulation and caspase-3 activity determination. PC12 cells were seeded into a 6 -well plate at a density of $1 \times 10^{5}$ cells/well. After differentiation, cells were pre-treated with $5 \mu \mathrm{g}$ and $20 \mu \mathrm{g}$ TL04 for $3 \mathrm{~h}$, followed by 12-h co-incubation with $20 \mathrm{mM}$ glutamate. Cultured medium was collected, and the intracellular LDH release was detected via in vitro Toxicology Assay kit (Sigma-Aldrich). Treated cells were lysed with radioimmunoprecipitation assay (RIPA) buffer (Sigma-Aldrich), intracellular ROS accumulation was measured by a ROS detection kit (Nanjing Biotechnology Co. Ltd., Nanjing, China), and caspase-3 activity was analyzed by a caspase-3 colorimetric detection kit (Enzo Life Sciences International, Inc.) according to the manufacturer's protocol.

Western blot analysis. DPC12 cells were pre-treated with $5 \mu \mathrm{g}$ and $20 \mu \mathrm{g}$ TL04 for $3 \mathrm{~h}$, followed by 24-h co-incubation with $20 \mathrm{mM}$ glutamate. Cells were lysed by RIPA buffer (Sigma-Aldrich) containing with $2 \%$ phenylmethanesulfonyl fluoride (Sigma-Aldrich) and 1\% protease inhibitor cocktail (Sigma-Aldrich). According to a previous study, cytoplasmic extracts were prepared for Cyto C release detection (25). After determination of protein concentration via the Bradford method using Coomassie Brilliant Blue G 250 (EMD Millipore), proteins were separated via 12\% sodium dodecyl sulfate-polyacrylamide gel electrophoresis gel and transferred electrophoretically onto nitrocellulose membranes (Bio Basic, Int., Amherst, NY, USA). The transferred membranes were then washed with Tris-buffered saline with $0.1 \%$ Tween-20 5 times every $5 \mathrm{~min}$, and blocked with $5 \%$ bovine serum albumin (Genview Scientific, Inc., El Monte, CA USA) for $3 \mathrm{~h}$ at room temperature. Then, the membranes were blotted with the following primary antibodies: Monoclonal rabbit Bcl-2 (ab32124), monoclonal rabbit Bax (ab32503), monoclonal mouse Cyto C (ab110325), polyclonal rabbit cleaved caspase-9 (ab2325) (all purchased from Abcam, Cambridge, UK), polyclonal rabbit cleaved caspase- 8 (AB1879), polyclonal rabbit cleaved caspase-3 (AB3623) (both purchased from EMD Millipore, Billerica, MA, USA) and polyclonal rabbit glyceraldehyde-3-phosphate dehydrogenase (GAPDH; sc-25778; Santa Cruz Biotechnology, Inc., Danvers, MA, USA) (all 1:1,000) at $4^{\circ} \mathrm{C}$ overnight, followed by incubation with horseradish peroxidase-conjugated mouse anti-rabbit IgG (sc-2357) and goat anti-mouse IgG (sc-2005) secondary antibodies (both 1:2,000; both purchased from Santa Cruz Biotechnology Inc.). Chemiluminescence was detected using enhanced chemiluminescence detection kits
(GE Healthcare). The intensity of the bands was quantified by scanning densitometry using software Image $\mathrm{J}$.

Statistical analysis. All data are presented as the mean \pm standard deviation. SPSS version 16.0 was used to perform all statistical analyses (IBM SPSS, Amronk, NY, USA). Data were evaluated by one-way analysis of variance to detect statistical significance, followed by post hoc multiple comparisons (Dunn's test). $\mathrm{P}<0.05$ was considered to indicate a statistically significant difference.

\section{Results}

Purification and characterization of polysaccharides purified from Tremella fuciformis. Anion exchange chromatography (DEAE-cellulose column) was performed to separate the crude polysaccharides obtained via water extraction (Fig. 1A). The samples were further purified by a gel permeation chromatography system Sepharose G-100, and an elution peak that appeared at 56 min was collected and named TL04 (Fig. 1B). Via calibration using the molecular weight curve, the molecular weight of TL04 was determined to be $2,033 \mathrm{kDa}$ (Fig. 1C). According the HPLC finger print, the percentage of rhamnose, mannose and glucose contained in TL04 was 1:5.04:1.87. The characteristic structures of TL04 were elucidated by FTIR spectra. A strong hydroxyl absorption peak $\left(3,400 \mathrm{~cm}^{-1}\right)$, a weak C-H absorption peak $\left(2,930 \mathrm{~cm}^{-1}\right)(26)$, and a $\mathrm{C}=\mathrm{O}$ characteristic absorption peak $\left(1,650 \mathrm{~cm}^{-1}\right)$ were observed (Fig. 1D). The absorption between 950-1,200 $\mathrm{cm}^{-1}$ indicated the existence a pyran ring skeleton structure within TL04 (27). The linkage mode of glucose present in TL0 4 was detected via Periodate oxidation-Smith degradation (Fig. 1E and F). After hydrolysis of TL04, L-rhamnose monohydrate, glucose, glycerol and erythritol were observed (Fig. 1F).

Effect of TL04 against glutamate-induced DPC12 cell damage. TL04 alone was not identified to affect DPC12 cell proliferation. Exposure to $20 \mathrm{mM}$ glutamate for $24 \mathrm{~h}$ resulted in $32.7 \%$ cell death $(\mathrm{P}<0.001)$; however, pretreatment with TL04 at 5 and $20 \mu \mathrm{g}$ strongly prevented the cell viability loss $(\mathrm{P}<0.05$ and $\mathrm{P}<0.01$, respectively; Fig. 2A). Pretreatment with $20 \mu \mathrm{g}$ TL04 for $3 \mathrm{~h}$ following incubation with $20 \mathrm{mM}$ glutamate for another $24 \mathrm{~h}$, significantly suppressed glutamate-enhanced LDH release was $(135.2 \pm 6.4$ vs. $111.3 \pm 3.6 \%$; $\mathrm{P}<0.01$; Fig. $2 \mathrm{~B})$. Furthermore, TL04 (5 and $20 \mu \mathrm{g}$ ) normalized the glutamate-induced over-accumulation of intracellular ROS $(165.3 \pm 4.4$ vs. $130.7 \pm 7.8$ and $111.3 \pm 3.6 \% ; \mathrm{P}<0.01$ and $\mathrm{P}<0.001$, respectively; Fig. $2 \mathrm{C}$ ) and hyper-activity of caspase-3 $(155.3 \pm 13.4$ vs. $120.7 \pm 10.8$ and $91.3 \pm 9.6 \%$; $\mathrm{P}<0.001$; Fig. 2D). In addition, results from Hoechst 33342 staining confirmed that TL0 4 markedly attenuated glutamate-induced apoptotic nuclei in DPC12 cells indicated by the reduction in the intensity of blue fluorescence (Fig. 2E).

TL04 restores the dissipation of MMP caused by glutamate. Results from JC-1 staining showed that TL04 significantly restored the dissipation of MMP indicated by the increment of red fluorescence emission (Fig. 3). Compared with the glutamate-treated cells, TL04 pretreatment at doses of 5 and $20 \mu \mathrm{g}$ significantly restored MMP up to $18.7 \pm 9.7$ and $24.9 \pm 7.9 \%$ $(\mathrm{P}<0.05$ and $\mathrm{P}<0.01$, respectively; Fig. 3). 
A

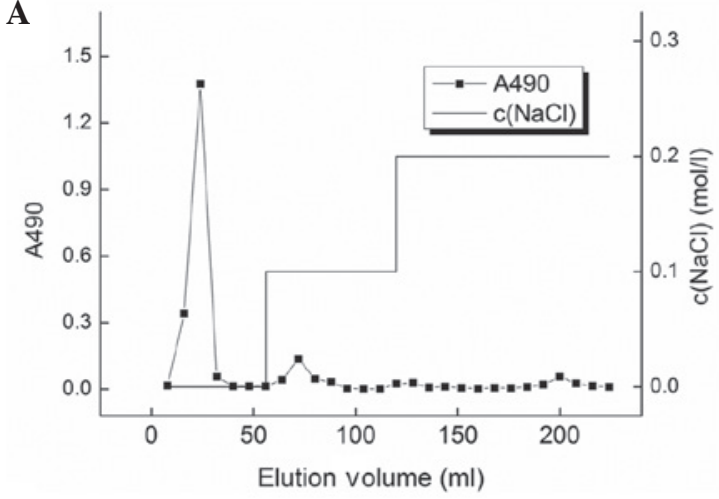

C

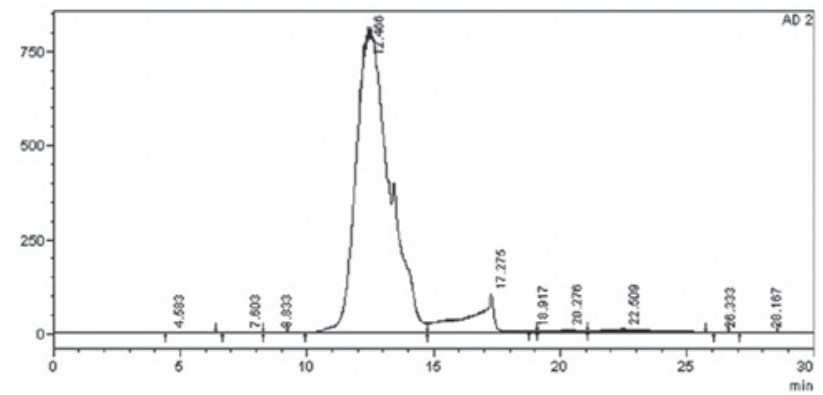

$\mathbf{E}$

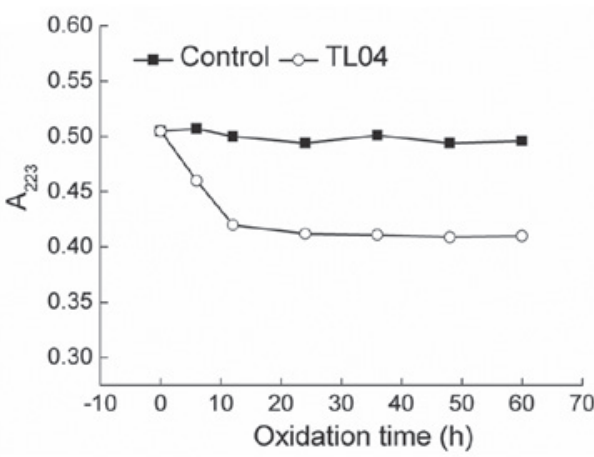

B

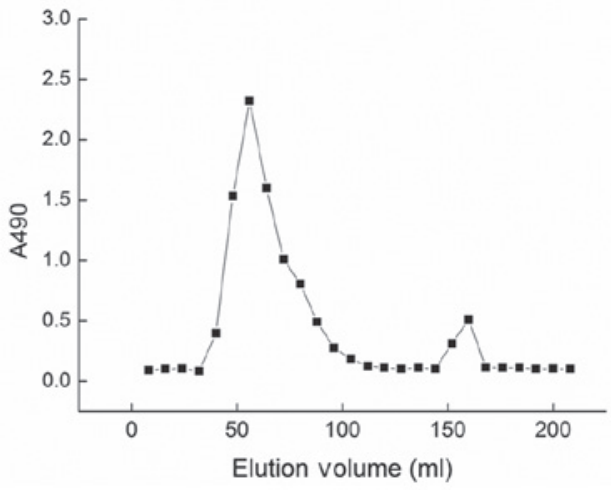

D

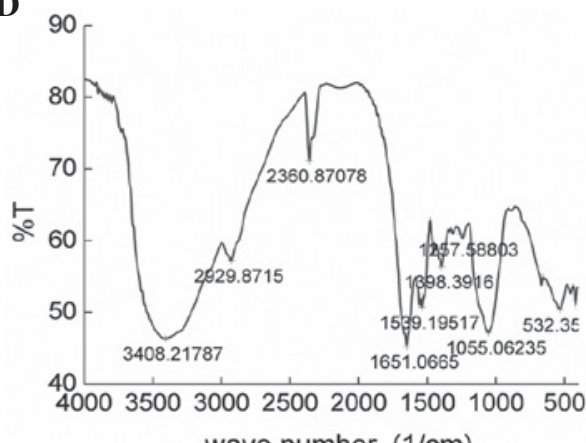

\section{F Smith degradation products}

\begin{tabular}{lccccc}
\hline Constituents & $\begin{array}{c}\text { L-rhamnose } \\
\text { monohydrate }\end{array}$ & Mannose & Glucose & Glycerol & Erythritol \\
\hline Before hydrolysis & - & - & - & - & - \\
Outside & + & - & + & + & + \\
Inside & - & - & - & - & - \\
\hline "+" Detected; "_" Undetected & & & & &
\end{tabular}

Figure 1. Purification and characterization of polysaccharides separated from Tremella fuciformis. (A) DEAE-Sepharose fast flow chromatogram of the crude polysaccharides, which was eluted using double distilled $\mathrm{H}_{2} \mathrm{O}$ at a flow rate of $1.0 \mathrm{ml} / \mathrm{ml}$. (B) The crude polysaccharides were further purified via Sepharose G-100. (C) Molecular weights of TL04 were determined by a HPLC/ELSD system equipped with a TSK-GEL G4000PWXL column. (D) Fourier transform infrared spectroscopy spectrum of TL04. (E) Periodate oxidation time course. (F) Products of Smith degradation of TL04 were detected using HPLC. HPLC, high performance liquid chromatography.

Effect of TLO4 on the expression of Bcl-2, Bax and Cyto C. Treatment with glutamate $(20 \mathrm{mM})$ resulted in a $21.1 \pm 6.3 \%$ reduction in the expression of $\mathrm{Bcl}-2$, a $19.3 \pm 3.8 \%$ increase in the expression of $\mathrm{Bax}$, and a $22.8 \pm 6.2 \%$ reduction in the level of cytoplasm Cyto $\mathrm{C}(\mathrm{P}<0.05$; Fig. 4). Pretreatment with $20 \mu \mathrm{g}$ TL04 restored the glutamate-reduced Bcl-2 level to $109.1 \pm 14.9 \%(\mathrm{P}<0.05)$, normalized glutamate-increased Bax expression to $98.3 \pm 4.4 \%(\mathrm{P}<0.01)$ and suppressed Cyto $\mathrm{C}$ release to $94.8 \pm 6.7 \%$ ( $\mathrm{P}<0.01$; Fig. 4$)$.

Caspase-dependent pathway contributes to TL04-mediated neuroprotection. Compared with control cells, the activity of caspase-8, caspase-9, and caspase-3 were enhanced to $118.9 \pm 4.4(\mathrm{P}<0.05), 121.3 \pm 3.8(\mathrm{P}<0.01)$ and $132.8 \pm 3.2 \%$ $(\mathrm{P}<0.01)$ in DPC12 cells exposed to $20 \mathrm{mM}$ glutamate for $24 \mathrm{~h}$ (Fig. 5). Pretreatment with $20 \mu \mathrm{g}$ TL0 4 decreased the levels of cleaved caspase- 8 , caspase- 9 and caspase- 3 to $101.1 \pm 5.4(\mathrm{P}<0.05), 98.3 \pm 4.3(\mathrm{P}<0.01)$ and $90.9 \pm 8.8 \%$
$(\mathrm{P}<0.01)$, respectively (Fig. 5). Furthermore, pretreatment with $10 \mu \mathrm{M}$ Ac-DEVD-CHO (a caspase-3 inhibitor) for $30 \mathrm{~min}$, and then co-incubation with or without TL04 for another $24 \mathrm{~h}$ significantly enhanced cell viability compared with glutamate-treated cells ( $\mathrm{P}<0.05$; Fig. 6).

\section{Discussion}

Recently, research on the structure and pharmacological activity of polysaccharides from Tremella fuciformis has received extensive attention $(20,28)$. The aim of the present study was to investigate the neuroprotective effect of the purified polysaccharide separated from Tremella fuciformis (TL04) against glutamate-induced DPC12 cell damage and the molecular mechanisms underlying these effects. The present results support the hypothesis that TL04 mediates neuroprotection of DPC12 cells indicated by the activity against glutamate-induced neurotoxicity on cell viability, 
A

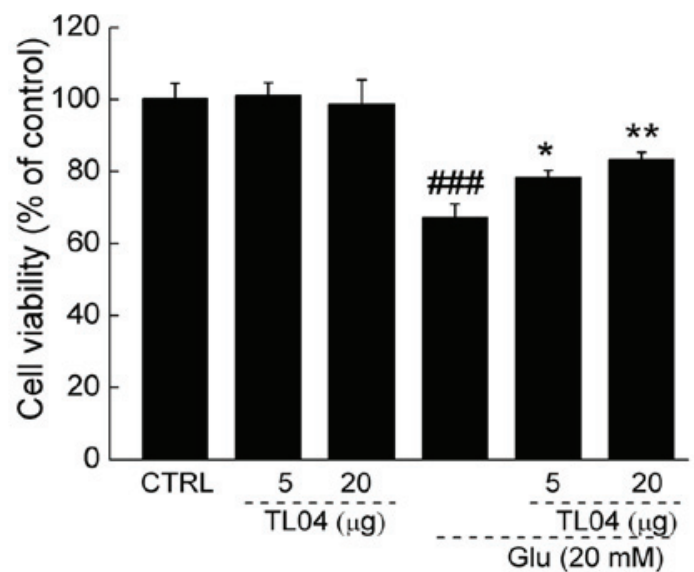

C

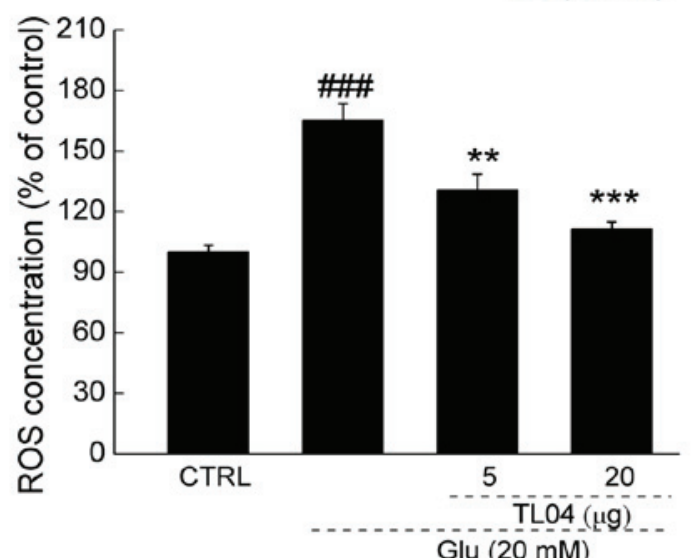

$\mathbf{E}$

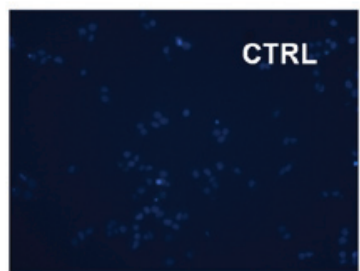

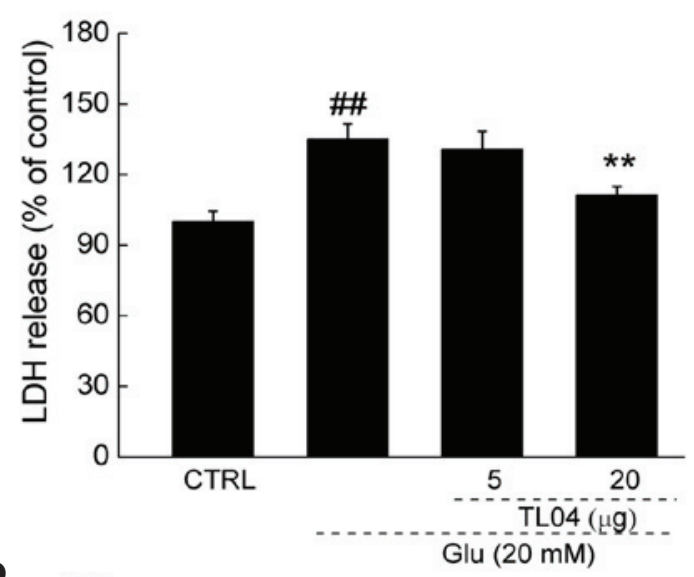

D
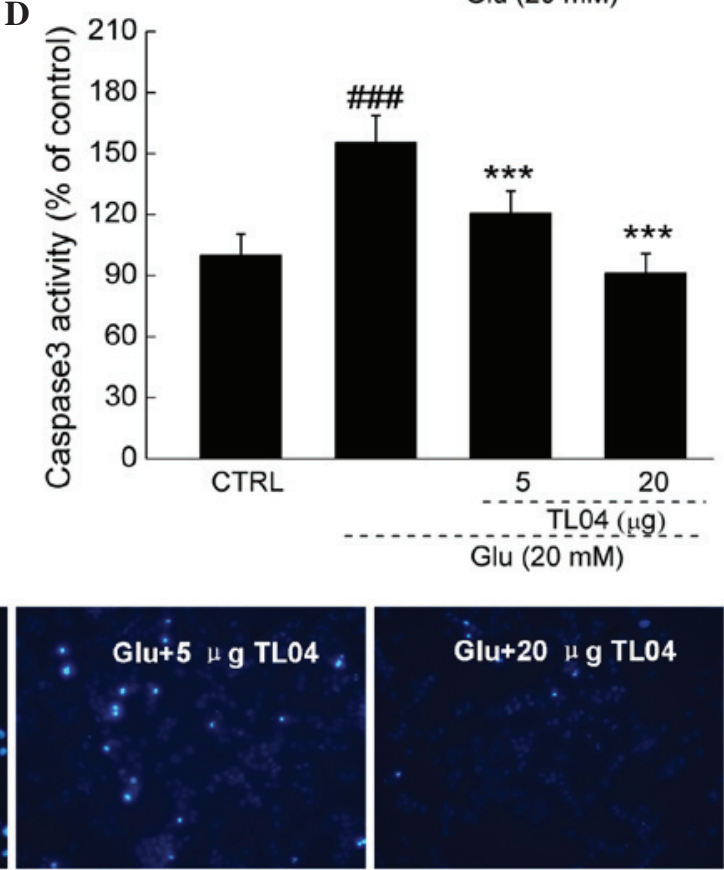

Figure 2. Effects of TL04 against Glu-induced neurotoxicity in DPC12 cells. Cells were pretreated with 5 and $20 \mu \mathrm{g}$ TL04 for 3 h, followed by exposure to $20 \mathrm{mM}$ glutamate for $24 \mathrm{~h}$. Compared with Glu-treated cells, TL04 pretreatment (A) enhanced cell viability, reduced (B) LDH release, (C) intracellular ROS accumulation, (D) caspase-3 activity and (E) apoptosis rate. Data are expressed as a percentage of corresponding control cells and the mean \pm standard deviation $(\mathrm{n}=6) .{ }^{\# \#} \mathrm{P}<0.01,{ }^{\# \# \#} \mathrm{P}<0.001$ vs. the untreated cells; ${ }^{*} \mathrm{P}<0.05,{ }^{* *} \mathrm{P}<0.01$ and ${ }^{* * *} \mathrm{P}<0.001$ vs. the Glu-exposed cells. Glu, glutamate; LDH, lactose dehydrogenase; ROS, reactive oxygen species; CTRL, control.
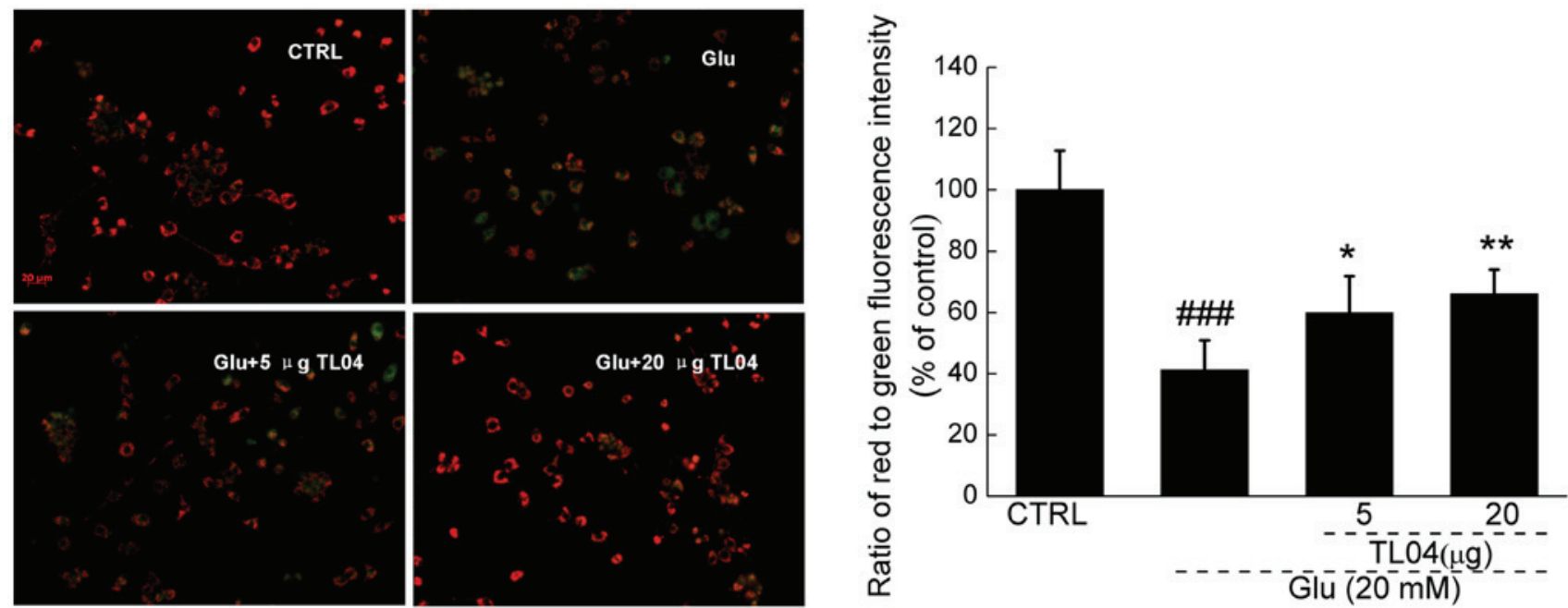

Figure 3. DPC12 cells were pretreated with 5 and $20 \mu \mathrm{g}$ TL04 for $3 \mathrm{~h}$, followed by exposure to $20 \mathrm{mM}$ glutamate for $12 \mathrm{~h}$, the effect of TL04 on mitochondrial dysfunction was determined via JC-1 staining (x20 objective; scale bar, $20 \mu \mathrm{m}$ ). Data are expressed as a percentage of corresponding control cells and the mean \pm standard deviation $(\mathrm{n}=3) .{ }^{\# \# "} \mathrm{P}<0.001$ vs. the untreated cells, ${ }^{*} \mathrm{P}<0.05$ and ${ }^{* *} \mathrm{P}<0.01$ vs. the Glu-exposed cells. CTRL, control; Glu, glutamate. 

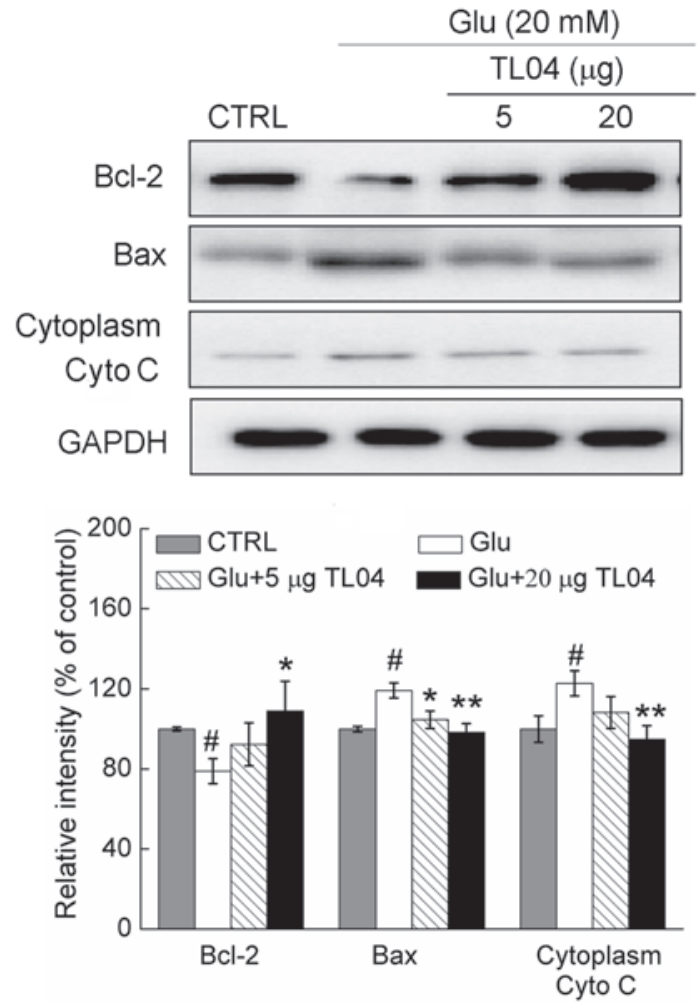

Figure 4. TL04 restored the abnormal expression of Bcl-2, Bax and Cyto C in the cytoplasm caused by glutamate after $24 \mathrm{~h}$ incubation in DPC12 cells. Data are expressed as a percentage of corresponding control cells and the mean \pm standard deviation $(\mathrm{n}=3) .{ }^{*} \mathrm{P}<0.05$ vs. the untreated cells; ${ }^{*} \mathrm{P}<0.05$ and ${ }^{* *} \mathrm{P}<0.01$ vs. the Glu-exposed cells. CTRL, control; GAPDH, glyceraldehyde 3-phosphate dehydrogenase; Cyto C, cytochrome $c$; Glu, glutamate.

ROS accumulation, LDH release, nuclear morphology, mitochondrial apoptotic alternation, and the expression of apoptotic proteins in DPC12 cells. Further data revealed that the caspase-dependent pathway is the predominant attribution for the neuroprotective activity of TL04.

Polysaccharides possess effective biological activities which are associated with their chemical structures. TL04, purified from Tremella fuciformis water extract, was characterized in the present study. The preliminary data from the ultraviolet spectrum suggested the absence of contamination of nucleic acid and proteins indicated by the lack of the absorbance peak at wavelengths of $260 \mathrm{~nm}$ and $280 \mathrm{~nm}$. The existence of $\mathrm{C}-\mathrm{H}, \mathrm{C}=\mathrm{O}, \mathrm{C}-\mathrm{O}-\mathrm{C}$ and $\mathrm{C}-\mathrm{O}-\mathrm{H}$ within TL04 was confirmed via FTIR spectra. Periodate oxidation-Smith degradation was applied to analyze the possible linkage of monosaccharides within TL04. During 48-h oxidation, little formic acid was produced suggesting inexistence of $1 \rightarrow$ and/or $(1 \rightarrow 6)$ linkage within TL04. The consumption of $<1$ mol periodate $(0.14 \mathrm{~mol}$ for TL04) indicated the existence of $(1 \rightarrow 3)$ linkage, and no glycerinum and erythritol were observed in the hydrolysate of TL04. Combining the data from permanganate oxidation, the backbone of TL04 is composed of $(1 \rightarrow 2)$ - and $(1 \rightarrow 4)$ linked-mannose and $(1 \rightarrow 3)$-linked-glucans.

Although a previous study indicated that Tremellafuciformis significantly promotes neurite outgrowth of $\mathrm{PC} 12$ cells (18), the present study focuses on the neuroprotective effect of TL04 against glutamate-induced toxicity in differentiated PC12 cells related to mitochondrial function. A series of experiments

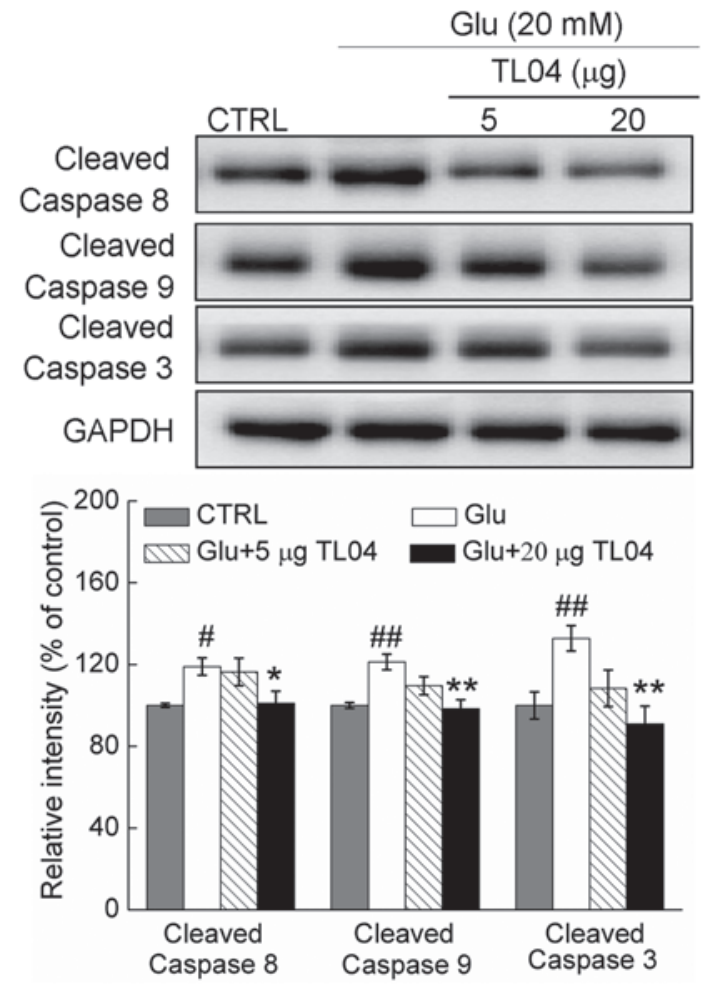

Figure 5. TL04 suppressed Glu-enhanced hyper-expression of cleaved caspase- 8 , caspase- 9 and caspase- 3 in DPC1 2 cells. Data are expressed as a percentage of corresponding control cells and the mean \pm standard deviation $(\mathrm{n}=3) .{ }^{\#} \mathrm{P}<0.05$ and ${ }^{\# \#} \mathrm{P}<0.01$ vs. the untreated cells; ${ }^{*} \mathrm{P}<0.05$ and ${ }^{* *} \mathrm{P}<0.01$ vs. the Glu-exposed cells.

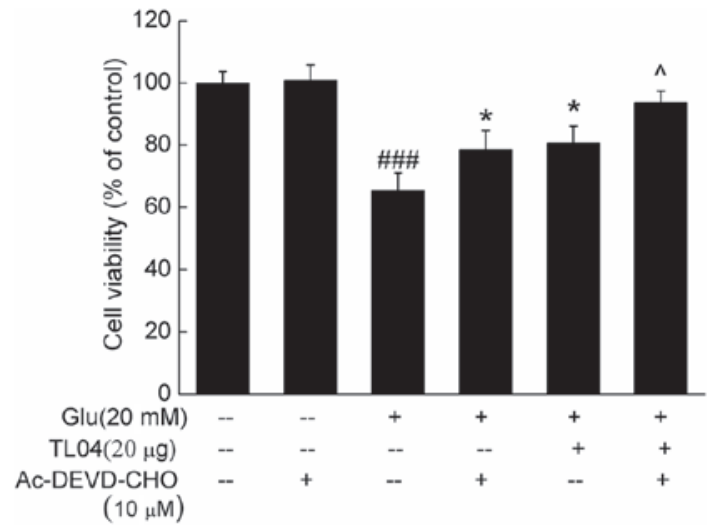

Figure 6. Ac-DEVD-CHO, a caspase-3 inhibitor, significantly enhanced the neuroprotective property of TL04 on cell viability. DPC12 cells were pretreated with $10 \mu \mathrm{M}$ Ac-DEVD-CHO for $30 \mathrm{~min}$, and exposed to 5 and $20 \mu \mathrm{g}$ TL0 4 for $3 \mathrm{~h}$, following by another $24 \mathrm{~h}$ co-incubation with $20 \mathrm{mM}$ Glu. Data are expressed as a percentage of corresponding control cells and the mean \pm standard deviation $(n=6)$. ${ }^{\# \# \#} \mathrm{P}<0.001$ vs. the control group; ${ }^{*} \mathrm{P}<0.01$ vs. the Glu-treated group; ${ }^{\wedge} \mathrm{P}<0.05$ vs. TL04-treated group. Glu, glutamate.

performed in differentiated PC12 cells supported the neuroprotection of TL04 against glutamate-induced toxicity in the present study. Mitochondrial function is considered to be key in cell apoptosis (29). The expression of Bcl-2 and Bax, located in the mitochondria, served as a hallmark to determine the levels of cell apoptosis and mitochondrial function (30). TL04 restored the dissipation of MMP, suppressed the expression of Bax, enhanced the level of $\mathrm{Bcl}-2$ and inhibited Cyto $\mathrm{C}$ release 
compared with glutamate-treated cells. It was previously demonstrated that when MMP dissipation was observed, the release of Cyto $\mathrm{C}$ from the mitochondria to the cytoplasm was enhanced (31). Moreover, TL04 successfully suppressed the glutamate-enhanced intracellular ROS level suggesting that ROS accumulation is responsible for mitochondrial membrane permeability (6,32). At physiologically low levels, ROS function as redox messengers in intracellular signaling and regulation, whereas excess ROS induce oxidative modification of cellular macromolecules, cause disruption of intracellular redox homeostasis, and is related to the opening of mitochondrial permeability transition pores (32). Thus the mitochondria-dependent apoptotic pathway was shown to be involved in the protective activity of TL04 against glutamate-associated neurotoxicity.

Caspases, a family of cysteine proteases, have been investigated as a mediator during neuronal apoptosis and neurodegeneration (33). Initiator caspases (caspase-8, -9 and -10) catalyze the proteolytic maturation of effector caspases (such as caspase-3, -6, -7), which result in cell death (34). The auto-catalytic activation of procasapase- 8 in the extrinsic apoptotic pathway further leads to the increase in mitochondrial membrane permeability $(31,35)$. Consequently, apoptotic stimuli trigger the release of mitochondrial intermembrane space proteins particularly Cyto C (36), which promotes caspase activation via forming a protein complex composed of Cyto C, Apaf-1 and caspase-9 (37). Activated caspase-9 in turn activates executioner caspase-3, which executes apoptosis (38). In the present study, TL04 suppressed the activation of caspase-8, caspase- 9 and caspase-3 in glutamate-treated DPC12 cells. Moreover, Ac-DEVD-CHO (a caspase-3 inhibitor) and TL04 co-treatment strongly enhanced cell viability compared with TL04-treated cells. These data suggest that TL04-mediated neuroprotection is associated with the caspase-dependent mitochondrial pathway.

In conclusion, the present study purified and characterized polysaccharides from Tremella fuciformis and confirmed the protective effect of TL04 against glutamate-induced neurotoxicity in DPC12 cells. In addition, the underlying mechanism was demonstrated to be associated with the caspase-dependent mitochondrial pathway. These findings raise the potential therapeutic application of Tremella fuciformis and purified polysaccharides TL04 against neurodegenerative diseases.

\section{Acknowledgements}

This study was supported by the National Science and Technology Support Program of P.R. China (grant no. 2012BAL29B05).

\section{References}

1. Traynelis SF, Wollmuth LP, McBain CJ, Menniti FS, Vance KM, Ogden KK, Hansen KB, Yuan H, Myers SJ and Dingledine R: Glutamate receptor ion channels: Structure, regulation and function. Pharmacol Rev 62: 405-496, 2010.

2. Reynolds IJ and Hastings TG: Glutamate induces the production of reactive oxygen species in cultured forebrain neurons following NMDA receptor activation. J Neurosci 15: 3318-3327, 1995.
3. Akaishi T, Nakazawa K, Sato K, Saito H, Ohno Y and Ito Y: Hydrogen peroxide modulates whole cell $\mathrm{Ca}^{2+}$ currents through L-type channels in cultured rat dentate granule cells. Neurosci Lett 356: 25-28, 2004.

4. Chamoun R, Suki D, Gopinath SP, Goodman JC and Robertson C: Role of extracellular glutamate measured by cerebral microdialysis in severe traumatic brain injury. J Neurosurg 113: 564-570, 2010.

5. Coyle JT and Puttfarcken P: Oxidative stress, glutamate and neurodegenerative disorders. Science 262: 689-695, 1993.

6. El-Najjar N, Chatila M, Moukadem H, Vuorela H, Ocker M, Gandesiri M, Schneider-Stock R and Gali-Muhtasib H: Reactive oxygen species mediate thymoquinone-induced apoptosis and activate ERK and JNK signaling. Apoptosis 15: 183-195, 2010.

7. Adams JM and Cory S: The Bcl-2 protein family: Arbiters of cell survival. Science 281: 1322-1326, 1998.

8. Tsujimoto Y and Shimizu S: Role of the mitochondrial membrane permeability transition in cell death. Apoptosis 12: 835-840, 2007.

9. Simon HU, Haj-Yehia A and Levi-Schaffer F: Role of reactive oxygen species (ROS) in apoptosis induction. Apoptosis 5: 415-418, 2000.

10. Ricci JE, Gottlieb RA and Green DR: Caspase-mediated loss of mitochondrial function and generation of reactive oxygen species during apoptosis. J Cell Biol 160: 65-75, 2003.

11. Methacanon P, Madla S, Kirtikara K and Prasitsil M: Structural elucidation of bioactive fungi-derived polymers. Carbohydrate Polymers 60: 199-203, 2005.

12. Zeng Y, Han Z, Qiu P, Zhou Z, Tang Y, Zhao Y, Zheng S, Xu C, Zhang X, Yin P, et al: Salinity-induced anti-angiogenesis activities and structural changes of the polysaccharides from cultured cordyceps militaris. PLoS One 9: e103880, 2014.

13. Habijanic J, Berovic M, Boh B, Plankl M and Wraber B: Submerged cultivation of Ganoderma lucidum and the effects of its polysaccharides on the production of human cytokines TNF- $\alpha$, IL-12, IFN- $\gamma$, IL-2, IL-4, IL-10 and IL-17. N Biotechnol 32: 85-95, 2015.

14. Ren M, Ye L, Hao X, Ren Z, Ren S, Xu K and Li J: Polysaccharides from Tricholoma matsutake and Lentinus edodes enhance 5-fluorouracil-mediated $\mathrm{H} 22$ cell growth inhibition. J Tradit Chin Med 34: 309-316, 2014.

15. Gao Q, Seljelid R, Chen H and Jiang R: Characterisation of acidic heteroglycans from Tremella fuciformis Berk with cytokine stimulating activity. Carbohydr Res 288: 135-142, 1996.

16. Ukai S, Kiriki H, Nagai K and Kiho T: Synthesis and antitumor activities of conjugates of mitomycin C-polysaccharide from Tremella fuciformis. Yakugaku Zasshi 112: 663-668, 1992 (In Japanese).

17. Ukai S, Kiho T, Hara C, Kuruma I and Tanaka Y: Polysaccharides in fungi. XIV. Anti-inflammatory effect of the polysaccharides from the fruit bodies of several fungi. J Pharmacobiodyn 6: 983-990, 1983.

18. Park HJ, Shim HS, Ahn YH, Kim KS, Park KJ, Choi WK, Ha HC, Kang JI, Kim TS, Yeo IH, et al: Tremella fuciformis enhances the neurite outgrowth of PC12 cells and restores trimethyltin-induced impairment of memory in rats via activation of CREB transcription and cholinergic systems. Behav Brain Res 229: 82-90, 2012.

19. Xu W, Shen X, Yang F, Han Y, Li R, Xue D and Jiang C: Protective effect of polysaccharides isolated from Tremella fuciformis against radiation-induced damage in mice. J Radiat Res 53: 353-360, 2012.

20. Zhang Z, Wang X, Zhao M and Qi H: Free-radical degradation by $\mathrm{Fe}^{2+} / \mathrm{Vc} / \mathrm{H}_{2} \mathrm{O}_{2}$ and antioxidant activity of polysaccharide from Tremella fuciformis. Carbohydr Polym 112: 578-582, 2014.

21. Du L, Song J, Wang H, Li P, Yang Z, Meng L, Meng F, Lu J and Teng L: Optimization of the fermentation medium for Paecilomyces tenuipes N45 using statistical approach. African Journal of Microbiology Research 6: 6130-6141,2012.

22. Yan H,Zhu D, Xu D, Wu J and Bian X: A study on Cordyceps militaris polysaccharide purification, composition and activity analysis. African Journal of Biotechnology 7: 4004-4009, 2008

23. Dong Y, Hu S, Liu C, Meng Q, Song J, Lu J, Cheng Y, Gao C, Liu Y, Wang D and Teng L: Purification of polysaccharides from Cordyceps militaris and their anti-hypoxic effect. Mol Med Rep 11: 1312-1317, 2015

24. Mosmann T: Rapid colorimetric assay for cellular growth and survival: Application to proliferation and cytotoxicity assays. J Immunol Methods 65: 55-63, 1983.

25. Yang CL, Chik SC, Li JC, Cheung BK and Lau AS: Identification of the bioactive constituent and its mechanisms of action in mediating the anti-inflammatory effects of black cohosh and related Cimicifuga species on human primary blood macrophages. J Med Chem 52: 6707-6715, 2009. 
26. Santhiya D, Subramanian S and Natarajan K: Surface chemical studies on sphalerite and galena using extracellular polysaccharides isolated from Bacillus polymyxa. J Colloid Interface Sci 256: 237-248, 2002.

27. Pielesz A: Vibrational spectroscopy and electrophoresis as a 'golden means' in monitoring of polysaccharides in medical plant and gels. Spectrochim Acta A Mol Biomol Spectrosc 93: 63-69, 2012 .

28. Du XJ,Zhang JS, Yang Y, Tang QJ, Jia W and Pan YJ: Purification, chemical modification and immunostimulating activity of polysaccharides from Tremella aurantialba fruit bodies. J Zhejiang Univ Sci B 11: 437-442, 2010.

29. LeeCS,Kim YJ,Lee MS,HanES and Lee SJ: 18beta-Glycyrrhetinic acid induces apoptotic cell death in $\mathrm{SiHa}$ cells and exhibits a synergistic effect against antibiotic anti-cancer drug toxicity. Life Sci 83: 481-489, 2008.

30. Raisova M, Hossini AM, Eberle J, Riebeling C, Wieder T, Sturm I, Daniel PT, Orfanos CE and Geilen CC: The Bax/Bcl-2 ratio determines the susceptibility of human melanoma cells to CD95/Fas-mediated apoptosis. J Invest Dermatol 117: 333-340, 2001.
31. Kroemer G, Dallaporta B and Resche-Rigon M: The mitochondrial death/life regulator in apoptosis and necrosis. Annu Rev Physiol 60: 619-642, 1998.

32. Circu ML and Aw TY: Reactive oxygen species, cellular redox systems and apoptosis. Free Radic Biol Med 48: 749-762, 2010.

33. Zhang $\mathrm{L}$ and Zhang S: Modulating Bcl-2 family proteins and caspase-3 in induction of apoptosis by paeoniflorin in human cervical cancer cells. Phytother Res 25: 1551-1557, 2011.

34. Hu Q, Wu D, Chen W, Yan Z and Shi Y: Proteolytic processing of the caspase-9 zymogen is required for apoptosome-mediated activation of caspase-9. J Biol Chem 288: 15142-15147, 2013.

35. Boatright KM, Renatus M, Scott FL, Sperandio S, Shin H, Pedersen IM, Ricci JE, Edris WA, Sutherlin DP, Green DR and Salvesen GS: A unified model for apical caspase activation. Mol Cell 11: 529-541, 2003.

36. Fulda S and Vucic D: Targeting IAP proteins for therapeutic intervention in cancer. Nat Rev Drug Discov 11: 109-124, 2012.

37. Yuan S and Akey CW: Apoptosome structure, assembly and procaspase activation. Structure 21: 501-515, 2013.

38. Jin Z and El-Deiry WS: Overview of cell death signaling pathways. Cancer Biol Ther 4: 139-163, 2005. 